\title{
APRENDIZAJE BASADO EN JUEGOS PARA LA ENSEÑANZA DE LA PROGRAMAGIÓN ORIENTADA A OBJETOS Y EL DISEÑO MULTIMEDIA
}

\author{
LEARNING BASED ON GAMES FOR TEACHING OBJECT \\ ORIENTED PROGRAMMING AND MULTIMEDIA DESIGN
}

\section{Giovanni Norero Ochoa}

Técnico en infografía e Ingeniero en Diseño Gráfico de nacionalidad ítalo-ecuatoriana. Magíster en Diseño y Gestión de Marcas, graduado de la Escuela Superior Politécnica del Litoral, cursa actualmente un programa doctoral en Humanidades y Artes en la Universidad Nacional de Rosario en la República de Argentina. Trabaja como profesor investigador para la Facultad de Comunicación Social de la Universidad de Guayaquil, en donde realiza investigaciones acerca de temas relacionados con la calidad integral y la gestión universitaria.

giovanni.noreroo@ug.edu.ec

orcid.org/0000-0003-2710-3950

Fecha de recepción: 12 de 0ctubre, 2017 / Aceptación: 5 de diciembre, 2017. 


\section{Resumen}

La educación tradicional suele resultar monótona 0 aburrida cuando trata temas que no se logran entender con facilidad o que no son del interés de los estudiantes. Debido a esto, los docentes se ven conminados a recurrir a métodos que logren cautivar la atención de sus alumnos y que los enganchen en el proceso de enseñanza. El aprendizaje basado en juegos forma parte de las tecnologías de la información y de la comunicación en la enseñanza, una metodología de trabajo cada vez más ampliamente utilizada en el ámbito de la educación superior. El presente trabajo recopila varias fuentes bibliográficas para que el lector pueda entender en qué consiste y cómo se aplica esta tecnología para crear un juego que ayude en la enseñanza de una materia de programación dirigido a aquellas personas que no cuentan con conocimientos informáticos.

Palabras clave: aprendizaje basado en juegos, diseño, juegos serios, programación, TIC.

\section{Abstract}

The present article is based on a historical analysis of emblematic figures in the fields of philosophy, mathematics, and engineering, this article proposes an updated reading of the ancestral origin of digital technology, and its intrinsic link with art and design. The works analyzed in this article include the Second Century BC. and XIV a.C. Initially. the clepsydra is presented as the basis for the elaboration of more sophisticated machines, such as the automata which is an ancestor of the current computers, and other devices. To demonstrate this point, the works of three notable inventors were examined: Archimedes of Syracuse, Ismail Al-Jazari and Leonardo Da Vinci.

Keywords: design, game based learning, ICT, programming, serious games. 
Introducción

Me lo contaron y lo olvidé. Lo vi y lo entendí. Lo hice y lo aprendí. Confucio.

Más de 2000 años han transcurrido desde que Platón instauró la academia y desde esa época hasta la actualidad la educación ha mantenido un formato parecido: el maestro habla/educa y el estudiante escucha/aprende. Tradicionalmente, en la formación elemental o preescolar, los profesores se han valido de medios interactivos y lúdicos para transmitir conocimientos de manera inconsciente a los niños y esto se ha logrado mediante la puesta en ejecución de actividades recreativas en las que se fomenta la participación inclusiva y analítica para desarrollar sus habilidades. Pero a medida que el niño se transforma en adolescente y luego en adulto esta práctica lúdica se pierde, la enseñanza se basa en técnicas teóricas o prácticas que muchas veces resultan ser monótonas y repetitivas para el estudiante. La principal desventaja de este tipo de instrucción recae en la facilidad con que los estudiantes están propensos a olvidar los contenidos que han tratado en el aula y esto se debe a que el docente expone su clase teórica mientras los alumnos se limitan a escuchar, muchas veces aburridos y con distracciones en el camino. Si la clase es práctica, se deben replicar las actividades que han sido explicadas, mas no siempre se incorporan elementos que generen un aprendizaje inductivo - deductivo que ayude a que el estudiante desarrolle sus habilidades cognitivas.

Desde sus inicios, la educación se ha valido de la tecnología para cubrir las necesidades de las sociedades contemporáneas. En un principio, se utilizaron los libros a los que se acudía en búsqueda de información, luego a parecieron los pizarrones, empleados para expresar mejor las ideas, las computadoras con sus proyectores y parlantes incorporaron imágenes y sonidos a las clases, y en la actualidad el uso de la Internet y los dispositivos móviles han creado una revolución en todos los sectores incluyendo la educación. En una época de cambios constantes y adelantos tecnológicos, la innovación y modernización son ideas que van de la mano, por esto resulta imperativo que la educación superior se valga de las tecnologías de la información y de la comunicación (TIC) para adoptar técnicas pedagógicas que ayuden a mejorar los procesos académicos y cumplir con las demandas de la sociedad del conocimiento actual.

El presente artículo expone los beneficios que brinda la implementación del aprendizaje basado en juegos, contrastándolos con la enseñanza tradicional y, además, demuestra cómo este método está siendo cada vez más utilizado en los procesos instructivos y en la capacitación de adultos no solo a nivel universitario, sino también profesional. La finalidad de este trabajo consiste en explorar esta técnica para brindar al lector un mejor entendimiento acerca del tema, a la vez que se aportan criterios que indican cómo se puede crear un juego serio para mejorar el proceso formativo en los estudiantes que cursan la asignatura de Programación Orientada a Objetos, de modo que estos nuevos conocimientos se empleen en el Diseño Multimedia.

Aprendizaje basado en juegos: ¿qué es y cómo funciona?

Históricamente, los juegos han ocupado un lugar sustancial en la vida de niños, adolescentes y hasta de adultos; todavía es común observar que en la educación preescolar se utilicen estas técnicas lúdicas para transmitir el conocimiento. Al descomponer los elementos divertidos en un juego inmediatamente queda al descubierto que lo que lo hace atractivo es el proceso de aprendizaje que lleva consigo (Trybus, 2014). En un juego, progresar cons- 
tituye un sinónimo de aprender, por esto, cuando una persona está inmersa en su interacción, su mente experimenta una serie de nuevas prácticas que sirven para entender el nuevo sistema, un principio atribuible a todos los juegos, ya sean recreativos 0 educativos.

El aprendizaje basado en juegos consiste en transmitir conocimientos a niños, adolescentes 0 adultos mediante el uso de videojuegos. En la educación inicial esta metodología es ampliamente utilizada, pero no ha gozado una buena acogida en la educación superior debido a la connotación negativa que los juegos de video para adultos suponen en la sociedad, se los relaciona con actos de violencia, soledad, adicciones, problemas de comunicación y comportamiento agresivo (Kowert, Vogelgesan, Festl, y Quandt, 2015). Sin embargo, en la última década se está evidenciando un cambio cultural en el que los videojuegos están siendo reprogramados y usados para brindar una experiencia educativa más envolvente y personalizada. Estudios sobre psicología cognitiva demuestran el valor del juego como potenciador del aprendizaje y de la adquisición de conocimientos, a la vez que contribuyen al desarrollo de los participantes en el plano intelectual-cognitivo, volitivo- conductual y afectivo-emocional (Smile Pill Marketing, 2014).

Desde la formación de la academia hasta los tiempos actuales, la educación básicamente se ha mantenido invariable con la exposición de sus clases magistrales en las que se presenta al estudiante una vasta cantidad de información a la que él tendrá que acudir para que pueda formar su propio criterio. El principal problema de esta metodología tradicional radica en que muchas veces carece de la personalización y de los procesos interactivos que ayudan a poner en un mejor contexto lo que se está explicando a lo que hay que sumarle el hecho de que el profesor prepara un solo contenido para todos los estudiantes de una misma clase en la que todos aprenden a ritmos diferentes, por lo tanto, si se avanza muy rápido, habrá alumnos que se podrán quedar rezagados y, si se avanza muy lento, aquellos que ya entendieron la clase llegarán a aburrirse. El aprendizaje no debe ser visto como un proceso monótono, impositivo o de memorización, sino como la entrega de herramientas y saberes que van a permitir que una persona sepa tomar la mejor decisión para que pueda actuar frente a una situación particular. Es ahí donde entra en acción el aprendizaje basado en juegos. Su función principal consiste en crear experiencias interactivas que motiven y enganchen a los estudiantes en el proceso de aprendizaje.

\section{De los juegos tradicionales a los juegos digitales}

Gracias a la tecnología actual, la mayoría de juegos tradicionales, conocidos también como los juegos de mesa, están siendo reemplazados por versiones digitales o por otros cuyas mecánicas son completamente diferentes y se desarrollan en entornos que demandan más tiempo e interacción del jugador. Por lo dicho, cada vez se escucha con mayor frecuencia que se incrementan las cifras de jugadores a nivel mundial al igual que las horas invertidas en dicha actividad. Según el Entertainment Software Association ${ }^{1}$ (2016), en los Estados Unidos el número de gamers o jugadores al finalizar el año 2016 se incrementó con respecto al año anterior y en ese mismo año el $63 \%$ de los hogares en este país contó con al menos una persona que utiliza videojuegos de manera regular. Esto significa un mínimo de 3 horas de interacción por semana y con tendencia a que estos valores se incrementen en el presente año. El estudio también reveló que la mayor cantidad de jugadores corresponden a los adultos jóvenes (18 35 años), un rango de edad que incluye a personas que asisten a universidades u otras instituciones de educación superior. 
Figura 1. Rango de edades de los videojugadores, Entertainment Software Association

(Norero, 2016).

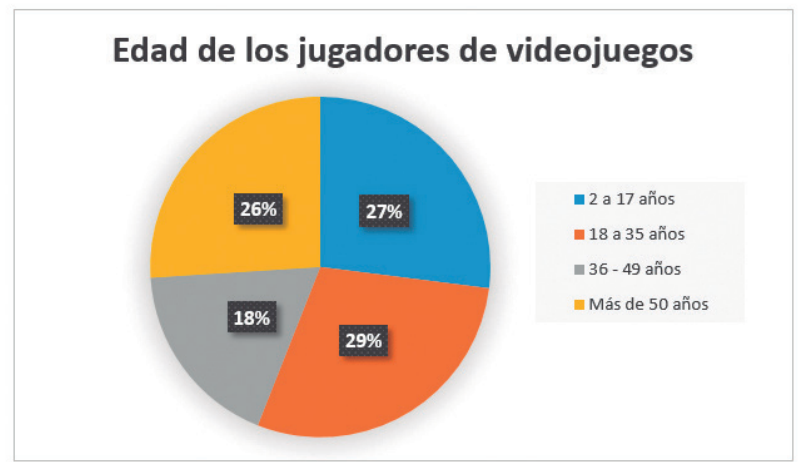

Presky, (citado por Chang, Liang, Chou y Lin, 2017) descompone los elementos que conforman un videojuego tradicional en metas, reglas, diversión, conflictos, desafíos, interacción social e historia. Es decir, detrás de un juego bien diseñado existe una serie de aprendizajes específicos que se deben llegar a dominar, y si a esto se le agrega la posibilidad de monitorear el progreso individual, se motiva al jugador a que obtenga un mejor resultado y se supere cada vez que lleva a cabo un nuevo intento. Un factor primordial de los videojuegos es que proporcionan un entorno rico de experimentación en primera persona en el que el jugador interactúa con el contexto creado, toma decisiones y percibe inmediatamente las consecuencias (Gros, 2009).

\section{Los juegos serios}

Los juegos serios consisten, como su nombre lo indica, en juegos orientados a actividades de enseñanza, como por ejemplo pilotar un avión comercial, emplear técnicas quirúrgicas en un caso médico, practicar la mezcla de gases para evitar una combustión química, tomar decisiones relacionadas con situaciones administrativas o económicas, entre otros. Ha recibido el nombre de serious games (juegos serios) debido a su naturaleza didáctica, su objetivo principal no se centra en la diversión, sino en la formación y práctica de habilidades, pues le permiten al usuario experimentar de forma segura y con medios simulados que no generan consecuencias peligrosas en la vida real, pero que aportan ex- periencia al jugador. El hecho de trabajar en un ambiente virtual da lugar a que se sienta familiar con el tema que se está tratando, lo que contrasta con el hecho de estar sentado en un salón de clase; de ese modo se establece una conexión más rápida entre el aprendizaje y la utilización de estos conocimientos en un caso verdadero. Muchos expertos en el aprendizaje basado en juegos utilizan los principios de diseño y flujo que dan lugar a que un videojuego se vuelva altamente adictivo. De igual forma, podemos anotar que existen cinco elementos básicos que aseguran la efectividad de un serious game, de acuerdo con el sitio web GameLearn (2017) estos son: historia, dinámica de interacción, retroalimentación, simulación y aprendizaje.

La historia supone un factor importante porque consigue que la persona se identifique con la temática del juego. Aunque no resulta imprescindible, sirve para crear un sentido de pertenencia con el que se logrará involucrar con mayor facilidad al jugador, que se verá más motivado a progresar en el juego hasta llegar a completarlo.

La dinámica de interacción establece cómo se va a llevar a cabo el juego; encierra las reglas, los objetivos, el sistema de recompensas, la clasificación de los jugadores y la interacción social presente. Los componentes sociales forman una parte fundamental en este tipo de juegos debido a que fomentan la competencia entre los diferentes jugadores. Para que una persona pueda superar o lograr una mejor puntuación que otra la primera deberá invertir más tiempo para dominar las mecánicas del juego y ob- 
tener en el proceso los saberes que el serious game intenta transmitir.

La retroalimentación o feedback personalizado instantáneamente le permite conocer al usuario no solo si las decisiones que toma dentro del juego poseen efectos positivos o negativos, también puede presentársele información detallada acerca de las razones por las cuales dicha decisión fue 0 no acertada de tal manera que el jugador puede ir estableciendo un patrón de conducta con el que podrá guiarse a futuro sabiendo las consecuencias que cierto comportamiento podría desencadenar.

La simulación sirve para poder representar un mundo virtual con el que el usuario se llegue a sentir identificado y así este pueda establecer una relación que permita asociar los conocimientos adquiridos en el juego con la cotidianidad. Por otro lado, la simulación en un serious game brinda la oportunidad de contar con ambientes seguros para tomar decisiones o probar escenarios que no repercutan en la vida real y ayuda a que los jugadores pierdan el miedo a realizar ciertas acciones que podrían traer consecuencias fatales o podrían resultar muy difíciles de intentar por primera vez en persona.

Si bien estos cuatro elementos mencionados forman parte de la estructura fundamental de la mayoría de videojuegos, el quinto elemento, aprendizaje, separa a un juego serio de un juego de mero entretenimiento. Esto no quiere decir que los juegos didácticos no pueden poseer un componente de diversión, sino que la diversión no es su prioridad, la enseñanza sí.

La Junta para la Clasificación del Software de Entretenimiento (ESRB por sus siglas en inglés), ente autorregulado y sin fines de lucro creado en el año 1994 en los Estados Unidos, se encarga de analizar el contenido, los elementos interactivos y la edad del público objetivo de cada videojuego para poder puntuarlo y clasificarlo, de ese modo los usuarios pueden saber qué tipo de juego es y a quiénes está dirigido antes de adquirirlo. Aunque la organización se creó en Norteamérica, la clasificación que emite sobre cada videojuego se respeta a nivel mundial debido a que cuenta con el aval de la Asociación de
Software de Entretenimiento. No obstante, al hablar sobre los juegos serios se torna necesario mencionar que todavía no existe un organismo oficial que se encargue de la regulación y clasificación de los contenidos, si bien varios estudios coinciden en una clasificación general conformada por las cinco categorías que mencionan Michaud, Álvarez, Álvarez y Djaouti (2012): salud, defensa y seguridad civil, educación, comunicación e información pública y capacitación profesional.

Los juegos serios de la categoría salud fueron los primeros en desarrollarse y son los que se han utilizado más ampliamente; existen más de 107 títulos como lo demuestra un estudio realizado por Wattanasoontorn, Boada, García, y Sbert (2013). Dentro de esta clasificación se pueden observar una gran variedad de juegos: algunos sirven para instruir a los pacientes para que sepan cómo tienen que actuar frente a algún proceso de recuperación, otros se dedican a crear hábitos saludables y, los más utilizados, son los simuladores en los que se observan síntomas y se practican procedimientos quirúrgicos en pacientes virtuales. Pulse!! consiste en un juego de simulación en primera persona. El jugador ve a través de los ojos de un doctor que se encuentra desempeñando sus labores en una sala de cuidados intensivos y en la que existe un paciente tendido en una Camilla. El doctor debe analizar los signos vitales del enfermo para solicitar los análisis sanguíneos respectivos y los exámenes para deducir las complicaciones que lo aquejan. En el camino, el jugador debe escoger la medicina y la dosis que debe administrar, debe dar órdenes a los enfermeros, analizar el resultado de los exámenes y tomar decisiones que ayuden a salvar la vida del paciente. Este juego no solamente es practicado por estudiantes, sino también por profesionales médicos que buscan mantenerse actualizados en conocimientos y procedimientos de esta naturaleza.

Pero los simuladores han ganado su prestigiosa reputación no solamente en el sector de la salud, sino también en área de la defensa y la seguridad civil. Así, el Departamento de Defensa de los Estados Unidos invierte grandes sumas de dinero en 
el desarrollo de serious games. En este contexto se creó el juego llamado Ambiente de Combate Interactivo Virtual (VICE, por sus siglas en inglés). Está diseñado para entrenar las habilidades cognitivas requeridas por el personal militar, seguridad interna y fuerzas policiales en la confrontación y resolución de problemas potenciales y actuales en ambientes urbanos, suburbanos y rurales (Dynamic Animation Systems, Inc., 2017). VICE utiliza el arsenal actualizado de los departamentos de defensa para contar con equipamiento y armas reglamentarias en ambientes en tres dimensiones, cuenta con dispositivos de comunicación para que varios jugadores puedan estar interconectados y jugando al mismo tiempo y de esta manera puedan funcionar como un equipo que incluso emplea tácticas para incrementar las probabilidades de éxito de una misión verdadera.

En la categoría de educación existe una muy variada gama de juegos serios que van desde aplicaciones usadas dentro del aula de clase para reforzar la explicación de un tema particular hasta juegos que pueden ser descargados en los dispositivos móviles para realizar un aprendizaje autónomo en cualquier momento y en cualquier lugar. Perfect World es un ejemplo del primer caso. Su temática está relacionada con el ciclo acuático, como parte del pensum de las Ciencias del Agua. En el juego, los estudiantes tratan de desplazar una molécula desde un elemento hacia el siguiente demostrando cómo el agua se mueve en su ambiente natural, al tiempo que ayuda a entender términos meteorológicos y conceptos que no pueden enseñarse mediante una clase teórica.

Si bien lo más común es encontrar juegos que sirvan para que el estudiante adquiera conocimientos, existen también otros que están dirigidos hacia los profesores. Simschool es un juego que nació hace más de 10 años como un proyecto de investigación de la Universidad de North Texas y la Universidad de Vermont. Su objetivo consiste en enseñar a educar mediante la implementación de un aula virtual en la que el docente interactúa con diferentes tipos de estudiantes en diversas situaciones. Este juego serio ayuda a incrementar el nivel de confianza de los profesores, al mismo tiempo que fomenta el uso de la tecnología y la alfabetización digital, mejora la puesta en ejecución de estrategias educativas, fortalece las técnicas de administración de estudiantes y brinda un mejor entendimiento acerca de las diferentes necesidades requeridas por los alumnos. Su fortaleza reside en que permite crear más de 10 trillones diferentes de combinaciones de características personales al mezclar género, raza, comportamiento y necesidades: los perfiles de cada estudiante puedan ser tan variados como en la realidad. Las técnicas académicas y tareas que el profesor virtual utiliza en su clase permiten evaluar en tiempo real la efectividad de los métodos de enseñanza suministrados.

En la categoría de información y comunicación pública, los juegos mayormente están orientados a crear conciencia para tratar de solucionar o minimizar problemas de índole social o cultural. Food Force, un juego creado por el Programa Mundial de Alimentos de las Naciones Unidas, tiene como fin instruir y promover la concienciación social de la lucha contra el hambre. La historia de este juego serio se desarrolla en una isla ficticia llamada Sheylan, ubicada en África. La isla está envuelta en una guerra civil que provoca sequía y escasez de alimentos, el objetivo del jugador consiste en formar parte de una comisión que ayude a alimentar a los millones de habitantes que sufren de hambruna. Durante el juego debe administrar las provisiones de comida que están por repartir para lograr que pueda durar hasta que llegue el siguiente cargamento y, además, intentará conseguir el apoyo de diferentes organizaciones que ayuden a la causa.

Al hablar de los juegos serios orientados para la capacitación profesional, se logra evidenciar una tendencia que demuestra que un gran número de juegos pertenecientes a esta categoría están dirigidos a incrementar la productividad dentro del ambiente laboral de una empresa 0 a mejorar las relaciones interpersonales. Pacific consiste en un juego de gestión de liderazgo y equipos en el que un grupo de náufragos deberán trabajar en conjunto para construir un globo que les ayude a abandonar 
la isla deshabitada en la que se encuentran. En el trayecto se presentan conflictos con las demás personas producto del estrés y de la desesperación por escapar. El grupo debe aprender y poner en práctica las habilidades para trabajar en equipo y bajo presión. Otro ejemplo es el juego Navieros, que está adaptado en la ciudad de Venecia de una Italia de la Edad Media. Consiste en valerse de técnicas de negociación, comunicación, planeamiento y servicio al cliente para administrar y ampliar la flota de barcos de una compañía. Este juego serio fue desarrollado específicamente por la compañía de electrónica de consumo LG para capacitar a sus empleados en España.

\section{Caso de estudio LG}

LG Electronics es una compañía que ocupa el puesto número 201 entre las 500 mejores empresas a nivel mundial (Fortune, 2017). Fundada en 1958 y asentada en Corea del Sur, posee 75 subsidiarias y da empleo a más de 83.000 personas en 119 países, en sus cuatro unidades de negocios dedicadas al entretenimiento del hogar, comunicaciones móviles, línea blanca y componentes automotrices (LG, 2017). El principio fundamental por el que se rige esta compañía es la innovación, presente no solo en la tecnología de sus productos, sino también en la interacción con sus clientes y en la formación de su personal. En el año 2014, LG España quiso hacer eco de la innovación que los caracteriza e incursionó en la capacitación de sus empleados mediante la elaboración de unas charlas informativas que culminaron con la ejecución de un juego serio llamado Navieros, que fue usado para formar 240 empleados de la empresa durante el periodo de un año. Debido a su locación geográfica y a su situación cultural, España es un país multinacional y multicultural, los ejecutivos en LG necesitaban una herramienta que sirva para poder llegar y capacitar a todos sus trabajadores y engancharlos en el proceso sin importar su nacionalidad, cultura o religión, por esto tomaron la decisión de optar por el aprendizaje basado en juegos.
El reporte presentado por GameLearn (2017), compañía desarrolladora del videojuego, muestra las necesidades que este juego debía cubrir:

- Entender y atender las principales necesidades y preocupaciones profesionales de los empleados para elevar su motivación.

- Seleccionar contenidos con un impacto directo en el trabajo de cada empleado y en su manera de interactuar con el cliente.

- Facilitar el autodesarrollo de la formación por parte del participante.

- Encontrar una solución formativa adaptada a la revolución digital del mercado.

Este juego serio se diseñó con seis capítulos. Antes de iniciar con el desarrollo de la historia, un mentor virtual se encargaba de exponer las clases magistrales con información complementaria que iba a ayudar al desarrollo del juego. El primer módulo estaba relacionado con la solución de conflictos con el cliente, los fundamentos de negociación y cómo evitar errores comunes. El módulo siguiente cubría las estrategias para obtener información y definir objetivos. El tercero se centraba en la flexibilidad y priorizaba los intereses grupales y no las posiciones personales. El cuarto hablaba sobre criterios y procedimientos para negociar y explicaba seis reglas claves para la gestión de cesiones. El quinto módulo trataba sobre la comunicación y las estrategias para anunciar y presentar una propuesta. El último módulo hacía una revisión de todo lo antes aprendido. El juego culminaba con la contratación de un capitán con el mejor perfil para cumplir con los requerimientos de la compañía naviera.

Según datos del mismo reporte, después de haber concluido con el programa de capacitación, se observó un nivel de satisfacción del juego de 9,8/10, un porcentaje de recomendación del 90\%, un alto nivel de aceptación, y que los participantes 
prefirieran volver a utilizar este método en el futuro. En cuanto a la efectividad del aprendizaje, el $97 \%$ (233) de los empleados que culminaron con el programa aseguraron que ponen en práctica lo aprendido y los porcentajes de satisfacción con respecto a las políticas de capacitación y el ambiente interno de la empresa se vio mejorado en un $18,64 \%$. También se observó un incremento de $12,61 \%$ en el nivel de productividad.

La empresa GameLearn se dedica a la creación de juegos serios orientados a la capacitación profesional; entre su portafolio de clientes constan más de 1000 compañías tales como Burger King, Adidas, Futisju, Bayer, Ford, Coca Cola, Hyundai y más. Crear un juego serio requiere de una gran inversión monetaria debido al desarrollo y programación requeridos en el proceso de producción. También existen universidades que se dedican a desarrollar proyectos para la creación de juegos aplicables a la educación media y superior con el afán de reducir los costos de elaboración e introducir características académicas específicas que integren estos juegos en el proceso de aprendizaje virtual.

\section{Creación de un juego serio para la educación}

Los procedimientos involucrados en la creación de un juego serio no difieren de aquellos empleados en algún otro proyecto. Si bien las herramientas de ejecución varían, todo trabajo debe iniciarse por un proceso de planificación en el que se establezcan las metas que se quieren alcanzar. En un juego de esta naturaleza, los objetivos se relacionan con los conocimientos que se intentan transmitir y que deben guardar relación con el syllabus de la asignatura o del área del conocimiento en el que se desea implementar el programa. Una vez analizados los objetivos, procedimientos y mecánicas del juego, se continúa con la etapa del diseño en la que se diseña el esqueleto del juego, esto es, el flujo u orden de aparición de las pantallas que se mostrarán al usuario final. Para que el juego sea llamativo, la historia y su desarrollo no deben ser lineales, sino variables, a fin de que cambien las decisiones que el jugador tome. Terminado el esqueleto, se programa el juego. Aquí se unen elementos visuales y sonoros hasta obtener un resultado tangible que pueda entrar en una etapa de implementación. Después de la creación, se pasa por una fase final de control de calidad como todos los programas, en la que se evalúa y prueba el juego para asegurar que no existan errores y que todo funcione como lo planificado.

Desarrollar un juego serio puede resultar una tarea muy complicada si se quiere partir desde cero, si no se cuentan con conocimientos acerca del diseño y la programación de herramientas informáticas. Por esto, el Departamento de Ingeniería del Software e Inteligencia Artificial de la Universidad Complutense de Madrid diseñó eAdventure, una plataforma que mezcla los aportes de expertos en los campos de la Ingeniería de Software, lenguajes formales de programación, pedagogía y tecnologías web con el fin de que las personas diseñen juegos serios incluso sin disponer de conocimientos informáticos avanzados como requeriría un proyecto de esta magnitud. Una de las ventajas de esta plataforma es su gratuidad, lo que facilita su adquisición y distribución. El único requerimiento existente consiste en descargar e instalar la plataforma en una computadora con sistema operativo Windows, Mac o Linux, con eso una persona puede empezar a crear sus propios juegos didácticos.

Al ejecutar el programa eAdventure, la ventana emergente va a solicitar la modalidad del juego serio que se va a crear y para lo cual se debe escoger entre dos posibles opciones: aventura en tercera persona que muestra al jugador que está siendo controlado, y aventura en primera persona, en la que se emplea un plano subjetivo que evidencia lo que el personaje virtual visualiza. Una vez dentro del editor de la aventura, el usuario deberá crear las escenas principales y secundarias; el protagonista, así como también los demás personajes y objetos con los que se va a poder interactuar; las conversaciones que se van a desarrollar dentro de la historia; las diferentes formas en que el juego va a evolucionar dependiendo de las decisiones tomadas $y$, finalmente, los perfiles de evaluación con los que 
se va a puntuar al jugador una vez concluido un capítulo o una sección particular. La plataforma cuenta con recursos adicionales que entregan información, ejemplos y videos que ayudan a entender cómo funciona este programa.

La enseñanza de la Programación Orientada a Objetos en una carrera de Diseño Gráfico supone un reto sustancial debido a que no todos los estudiantes poseen estudios informáticos, pero el dominio de dicha asignatura resulta cardinal para poder cursar las materias asociadas al diseño multimedia y a la programación web. Los métodos tradicionales no resultan suficientes para alguien sin experiencia en programación, por lo que el aprendizaje resulta ser un problema y no se lo puede lograr de una manera adecuada, por esto la creación de un juego serio se presenta como una alternativa idónea (Mitamura, Suzuki y Oohori, 2012). A continuación, describimos dos ideas aplicadas a la enseñanza de la Programación Orientada a Objetos, sin embargo, sus características específicas, así como su ejecución no se encuentran detalladas debido a que actualmente siguen en proceso de desarrollo por parte del autor del presente documento.

El tema principal del primer concepto se centra en los diagramas de flujo. El estilo de juego va a ser individual y la mecánica consiste en presentar una breve historia aleatoria que el jugador replicará mediante la selección de acciones dentro de un diagrama de flujo. A medida en que el usuario completa su diagrama, podrá ejecutarlo para verificar si las acciones se llevan a cabo de la misma manera en que la historia fue narrada. Al haber aprobado una historia, se presenta la siguiente que será más larga y más compleja. Este juego ayuda a identificar y familiarizarse con los símbolos de los diagramas de flujo y fomenta el desarrollo de la lógica usada en la programación.

El segundo concepto es un juego en el que participan dos personas y juegan en la modalidad por turnos. Se desarrolla en un tablero de ajedrez y cada jugador debe controlar la única pieza de su equipo; en cada turno, el jugador programa los movimientos de su pieza para poder acercarse a la pieza contraria y atacarla. En el camino, ambos deberán esquivar obstáculos o trampas que les impidan movilizarse 0 dejarlos vulnerables para un ataque por parte de su adversario. Este juego no solo aumenta las habilidades lógicas para entender cómo un objeto 0 robot debería moverse por su cuenta, sino que también requiere de planificación para evitar los obstáculos que se lleguen a presentar.

La Programación Orientada a Objetos es un prerrequisito para que los estudiantes puedan diseñar y desarrollar correctamente aplicaciones multimedia 0 web. Al decir diseño multimedia no nos referimos solo a la mezcla de texto, imágenes y audio, sino a la elaboración de contenido dinámico y rico en interacción con el usuario, por lo tanto, los conocimientos en programación se vuelven muy necesarios.

La implementación de estos juegos serios y de otras tecnologías de la información y de la comunicación en los ambientes educativos depende mucho de la predisposición que los docentes posean frente a la adopción de cambios y de nuevos métodos de enseñanza. Muchas veces la aplicación de innovaciones pedagógicas relacionadas con la tecnología trae consigo una barrera que se presenta en la falta de tiempo de los profesores para aprender y capacitarse y en las limitaciones económicas que el uso de nuevas tecnologías supone. Para lo último existen alternativas económicas y hasta gratuitas, pero para lo primero, solo se necesita una actitud positiva y eso depende de cada persona que esté dispuesta a superarse y a mejorar sus métodos académicos. 


\section{Conclusiones}

Las técnicas de enseñanza-aprendizaje deben ser actualizadas mediante la implementación de las tecnologías de la información y de la comunicación, para que los estudiantes se enganchen con la clase, esto porque la enseñanza tradicional puede resultar monótona y en algunos casos aburrida. El aprendizaje basado en juegos se constituye en una alternativa eficiente para lograr que los conocimientos lleguen y sean entendidos por más estudiantes. Los juegos calificados como serios ayudan al desarrollo de habilidades y al entendimiento acerca de temas que no pueden ser fácilmente explicados mediante una clase teórica. Por ese motivo, estos recursos están ganando cada vez mayor aceptación dentro del mundo académico, tanto que incluso desde el año 2012 ya se llevaron a cabo conferencias internacionales en donde se premiaron a los mejores juegos serios del año.

\section{Notas}

'La Asociación de Software de Entretenimiento (ESA) es una organización que realiza negocios, investigaciones de mercado y análisis acerca de temas como la protección de contenido global, propiedad intelectual y tecnología en favor de los publicadores de software interactivos. ESA representa a los intereses de la industria de videojuegos y desarrolladores de software a nivel federal y estatal en los Estados Unidos y otros países del mundo.

\section{Referencias bibliográficas}

Chang, C., Liang, C., Chou, P. y Lin, G. (2017). Is game-based learning better in flow experience and various types of cognitive load than non-game-based learning? Perspective from multimedia and media richness. Computers in Human Behavior, 218-227.

Dynamic Animation Systems, Inc. (2017). The Virtual Interactive Combat Environment. Recuperado de: http://www.d-a-s.com/VICE.

eAdventure. (2017). Acerca de. Recuperado de: http://e- adventure.e-ucm.es/about/

Entertainment Software Association. (2016). Essential facts about the computer and video game industry. Recuperado de:

http://essentialfacts.theesa.com/Essential-Facts-2016.pdf

Fortune. (2017). Global 500. Obtenido de Fortune http://fortune.com/global500/lg- electronics/

GameLearn. (2017a). Caso de éxito Gamificación LG. Recuperado de: https://www.game-learn.com/wp-content/uploads/2013/06/01_CS_LG_ESP.pdf

GameLearn. (2017b). Todo lo que necesitas saber sobre los serious games y el game- based learning, explicado con ejemplos. Recuperado de: https://www.game-learn.com/lo-que-necesitas-saber-serious-gamesgame-based-learning-ejemplos/

Gros, B. (2009). Certezas e interrogantes acerca del uso de los videojuegos para el aprendizaje. (E. U. Sevilla, Ed.) Revista Internacional de Comunicación Audiovisual, Publicidad y Literatura (7), 251-264. 
Kowert, R., Vogelgesan, J., Festl, R., y Quandt, T. (2015). Psychosocial causes and consequences of online video game play. Computers in Human Behavior, 51-58.

LG. (2017). Acerca de LG. Recuperado de: LG Electronics: http://www.Ig.com/ec/acerca- de-Ig/nuestra-marca

Michaud, L., Álvarez, J., Álvarez, V., y Djaouti, D. (2012). Serious Games: Issues, offer and market. Tercera edición. Montpellier: IDATE Consulting \& Research.

Mitamura, T., Suzuki, Y., y Oohori, T. (2012). Serious Games for Learning Programming Languages. 2012 IEEE International Conference on Systems, Man, and Cybernetics (SMC). Seúl, Corea del Sur: IEEE.

Smile Pill Marketing. (17 de octubre de 2014). El aprendizaje lúdico: una novedosa estrategia de capacitación. Recuperado de: Smile Pill Marketing: https://spmarketing.com/el-aprendizaje- ludico-como-estrategia-de-capacitacion/

Trybus, J. (2014). Game-Based Learning: What it is, Why it Works, and Where it's Going. Recuperado de: http://www.newmedia.org/game-based-learning- what-it-worksand-where-its-going.html.

Wattanasoontorn, V., Boada, I., García, R., y Sbert, M. (2013). Serious games for health. Entertainment Computing, 231-247.

\section{Figuras}

Figura 1. Norero, G. (2016). Rango de edades de los jugadores. Entertainment Software Association. 